\title{
Mechanisms of water infiltration into conical hydrophobic nanopores
}

\author{
Received 20th March 2009, Accepted 14th May 2009 \\ First published as an Advance Article on the web 12th June 2009 \\ DOI: $10.1039 / \mathbf{b} 905641 \mathrm{f}$
}

Ling Liu, ${ }^{a}$ Jianbing Zhao, ${ }^{a}$ Chun-Yang Yin, ${ }^{a b}$ Patricia J. Culligan ${ }^{a}$ and Xi Chen $* a$

Fluid channels with inclined solid walls (e.g. cone- and slit-shaped pores) have wide and promising applications in micro- and nano-engineering and science. In this paper, we use molecular dynamics (MD) simulations to investigate the mechanisms of water infiltration (adsorption) into cone-shaped nanopores made of a hydrophobic graphene sheet. When the apex angle is relatively small, an external pressure is required to initiate infiltration and the pressure should keep increasing in order to further advance the water front inside the nanopore. By enlarging the apex angle, the pressure required for sustaining infiltration can be effectively lowered. When the apex angle is sufficiently large, under ambient condition water can spontaneously infiltrate to a certain depth of the nanopore, after which an external pressure is still required to infiltrate more water molecules. The unusual involvement of both spontaneous and pressure-assisted infiltration mechanisms in the case of blunt nanocones, as well as other unique nanofluid characteristics, is explained by the Young's relation enriched with the size effects of surface tension and contact angle in the nanoscale confinement.

\section{Introduction}

In the past decade, nanofluidics ${ }^{1-3}$ has attracted significant interest owing to its strong relevance to biological transport, drug delivery, sensing, energy dissipation, conversion and storage, environmental science and engineering, etc. ${ }^{4-8}$ Two fundamental physical processes are generally involved, namely, the filtration (sorption) of fluids into nanopores and the transport of fluids inside nanopores. Both of them are closely associated with the unique molecular interactions between the solid and liquid phases, ${ }^{9}$ the structural $^{10}$ and energetic $^{11}$ differences between the bulk and confined states of the liquids, as well as the physical properties of solids (e.g. partial charge and surface roughness ${ }^{12-15}$ ) and liquids (e.g. ionization ${ }^{16,17}$ ).

Most previous studies of nanofluidics were limited to nanochannels with an invariant circular cross-section along the axial direction, e.g. carbon nanotubes (CNTs). ${ }^{18-21}$ Nanochannels with inclined solid walls, such as conical nanopores, are much less studied where the available literature was mainly focused on transport behavior. ${ }^{22}$ Note that conical pores with somewhat larger dimensions have been employed in many innovative ways, such as DNA sensing, ${ }^{23}$ ionic oscillation, ${ }^{24}$ particle trapping ${ }^{25}$ and pumped cooling loops, ${ }^{26}$ among others. To further expand such capabilities to nanofluidic devices, it is important to understand the fundamental nanofluid behaviors, including infiltration mechanisms, in conical nanochannels.

There are two distinctive geometrical factors contributing to the infiltration of nanofluids into conical nanopores. First, the

\footnotetext{
${ }^{a}$ Columbia Nanomechanics Research Center, School of Engineering and Applied Sciences, Mail Code 4709, Columbia University,

New York, NY 10027-6699,USA.E-mail: xc2107@columbia.edu;

Fax: + 1-212-854-6267; Tel: + 1-212-854-3787

${ }^{b}$ Faculty of Chemical Engineering, Universiti Teknologi MARA,

40450 Shah Alam, Selangor, Malaysia
}

inclination angle of the solid wall should play an important role, as it does at the continuum level. ${ }^{27,28}$ According to the classical Young's relation, the infiltration of water into tube-shaped capillaries (with invariant cross-section) requires an external pressure (usually termed as the infiltration pressure, $\left.P_{\text {in }}\right)$ when the solid phase is hydrophobic, $\dagger$ but can occur spontaneously $\left(P_{\text {in }} \leq 0\right)$ when the solid phase is hydrophilic. For capillaries with inclined walls, this infiltration pressure is determined not only by the contact angle, but also by the inclination angle of the solid wall. ${ }^{27,28}$ The competition between spontaneous infiltration and pressure-assisted infiltration is one of the main focuses of this paper.

Second, with the varying cross-section of a conical pore, the size effects of contributing variables (e.g. contact angle ${ }^{9}$ and surface tension) are varied as the fluid front advances, which may yield observations unique to the nanoscale. While the nanofluid behaviors are governed by established physics laws, e.g. the Young's relation for infiltration ${ }^{11}$ and Poiseuille's relation for transport, ${ }^{29}$ the size effects of system variables (molecular structure, ${ }^{30}$ contact angle, ${ }^{9}$ velocity profile ${ }^{29}$ and surface tension, among others) may strongly affect the physical processes. It is therefore important to understand the trend of those physical properties with respect to the characteristic length of the nanoscale confinement.

\section{Model and method}

In this study, we use molecular dynamics (MD) to explore useful insights into the complex behaviors of liquid infiltration into cone-shaped nanopores, in particular the profound yet fundamental influences of the inclination angle and varying pore size. The simulations are performed using the

$\dagger$ A solid phase is hydrophilic if a water droplet resting on a flat surface of the solid has a contact angle smaller than $90^{\circ}$. For a hydrophobic solid phase, the contact angle is larger than $90^{\circ}$. 


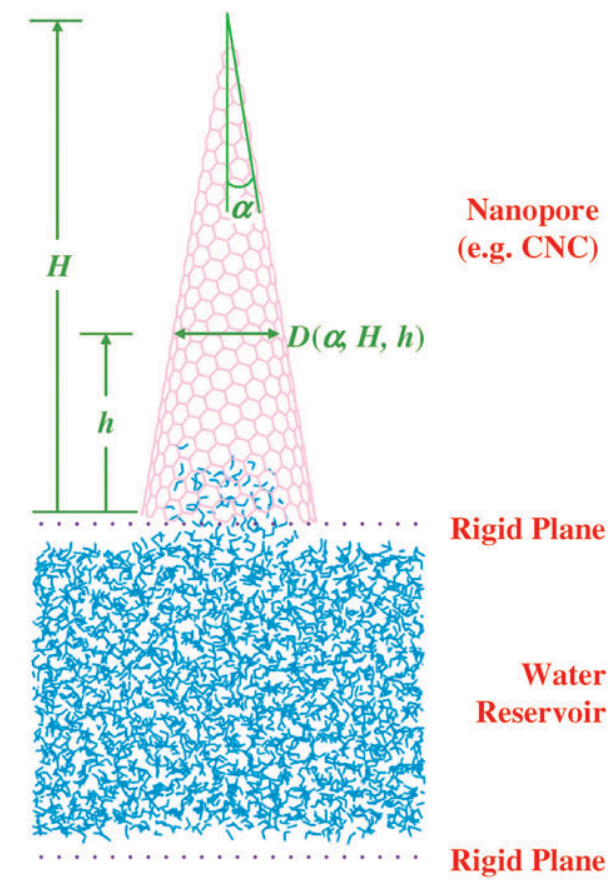

Fig. 1 The computational model. A carbon nanocone (CNC) characterized by $\alpha$ and $H$ is fixed in space, with its opening end immersed in a reservoir filled with water molecules. The upper rigid plane is fixed while the lower one is movable for adjusting the pressure inside the reservoir. A periodic boundary condition is applied to the four lateral faces of the computational cell.

LAMMPS $^{31}$ (large-scale atomic/molecular massively parallel simulator) package, with the NVT ensemble and the temperature fixed at $300 \mathrm{~K}$. Water is considered as the liquid phase and carbon nanocone ${ }^{32}$ (CNC) is adopted as a model structure of the cone-shaped nanopore. A carbon nanocone (Fig. 1), rolled up from a cropped graphene sheet, is characterized by two geometrical parameters, the half apex angle, $\alpha$, and the height, $H$. Four representative values of $\alpha$ are adopted $\left(9.6,19.5,30\right.$ and $\left.41.8^{\circ}\right)$ to account for a relatively large variation of the apex angle. CNTs of varying diameters are analyzed to represent the case with $\alpha=0^{\circ}$. Furthermore, different values of $H$ are also taken into account. In all these cases, the pore diameter, $D$, is adopted as the characteristic length, which, according to Fig. 1, is a function of the depth (distance from the pore opening), $h$, as well as $\alpha$ and $H$. The interatomic van der Waals interaction is described by the Lennard-Jones (LJ) empirical forcefield, $U(r)=$ $4 \varepsilon\left[(\sigma / r)^{12}-(\sigma / r)^{6}\right]$, where $r$ denotes the distance between atoms, and $\varepsilon$ and $\sigma$ are energy and length parameters, respectively. Water molecules are modeled by the rigid extended simple point charge potential $\mathrm{SPC} / \mathrm{E}^{33}$ while the carbonoxygen LJ parameters are extracted from the experimental low-coverage isotherm data of oxygen adsorption on graphite. ${ }^{34}$ By using these molecular models and parameters,

$\ddagger$ This system has been verified to provide consistent results as the size of computational cell is varied; that is, the computed results (e.g. water pressure) have negligible difference if more water molecules are involved in the system. the graphene used as the raw material for building CNTs and CNCs is ensured to be physically hydrophobic. ${ }^{9}$

Fig. 1 illustrates the computational model. A nanocone, assumed to be rigid, is fixed in space with its opening end immersed in a reservoir $(45 \times 45 \times 35 \AA)$ filled with the water phase. $\ddagger$ The top and bottom surfaces of the reservoir are bounded by two rigid planes, with the upper one fixed and the lower one movable to mimic a piston. A periodic boundary condition is imposed on the four lateral planes of the computational cell. Initially, the opening of the nanocone is covered by a rigid lid, and a specific number (about 2400) of water molecules are placed in the reservoir such that the pressure inside is close to zero (ambient) after equilibrium. Next, the lid is removed, and upon reaching the new equilibrium state, one could explore whether the liquid molecules could spontaneously infiltrate the nanocone without any external force applied. Further, as the piston is moved upwards and the reservoir volume is reduced, a relationship is measured between the elevated reservoir pressure and the number of infiltrated water molecules. The loading is carried out in a quasi-static manner, as the piston's position is held for a sufficiently long time after each loading increment to allow system equilibration. $\S$ The water pressure, $P$, is thereby evaluated for all loading steps according to the immediate density of water inside the reservoir, $\rho$, namely, $P=0.1+$ $298\left(\rho^{7.15}-1\right)$ where $P$ has the units MPa and $\rho$ is in units of $\mathrm{g} \mathrm{cm}^{-3} \cdot 35$

\section{Results and discussion}

Based on our MD results, a significant $\alpha$-dependency accompanying the nanofluid infiltration into cone-shaped nanopores is unveiled. At ambient condition (without external force), Fig. 2 shows the equilibrium positions of water front for various $\alpha$ (including a nanotube with $\alpha$ equals to zero). For nanocones with small apex angles, water molecules are expelled outside, suggesting that external assistance is needed to initiate infiltration. In the case of blunt nanocones, nevertheless, spontaneous infiltration occurs despite the hydrophobic nature of the solid phase, making the nanocones nominally hydrophilic. Interestingly, spontaneous infiltration does not wet the entire nanocone in these cases; the invaded water front stops at a particular height, which corresponds to a pore diameter of about $19 \AA$ for $\alpha=30^{\circ}$ and about $11.4 \AA$ for $\alpha=41.8^{\circ}$ (for all $H$ studied).

In all cases, further infiltration of water molecules is assisted by the external pressure imposed to the system. The effects of the geometrical constraints are evident when one compares the pressure-infiltration relationships between a nanotube and a nanocone. Fig. 3 plots the number of infiltrated water

§ System equilibrium in this study is defined as a sufficiently equilibrated state where the kinetic energy of water molecules inside the reservoir is quite uniform. It usually takes several hundreds of picoseconds to a few nanoseconds to regain the equilibrium after the piston is moved and fixed at a new position.

- A nanopore is nominally hydrophilic if water can spontaneously infiltrate the nanopore. When the cross-section of nanopore is invariant, the hydrophobicity (hydrophobic or hydrophilic nature) of a nanotube is determined by that of the solid phase. However, such correspondence breaks down for nanopores with varying cross-sections. 


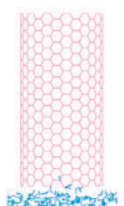

(a) $\alpha=0^{\circ}$

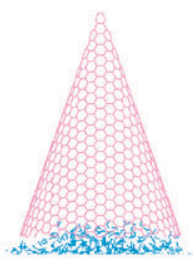

(b) $\alpha=19.5^{\circ}$

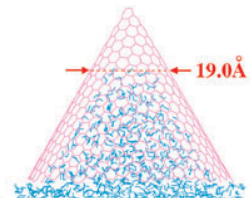

(c) $\alpha=30^{\circ}$

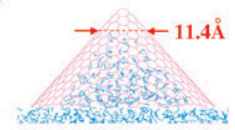

(d) $\alpha=41.8^{\circ}$
Fig. 2 The position of water front when no external pressure is applied. (a-b) Infiltration is prohibited for a $(10,10)$ CNT and a CNC with $\alpha=19.5^{\circ}$ (and similarly for all the other CNTs tested and for CNCs with smaller angles (not shown)). (c-d) Infiltration spontaneously initiates for the two CNCs with $\alpha$ equal to 30 and $41.8^{\circ}$. Note that, for blunt $\mathrm{CNCs}$, the water front could only access a critical pore diameter (regardless of $H$ ) as identified in the figure.

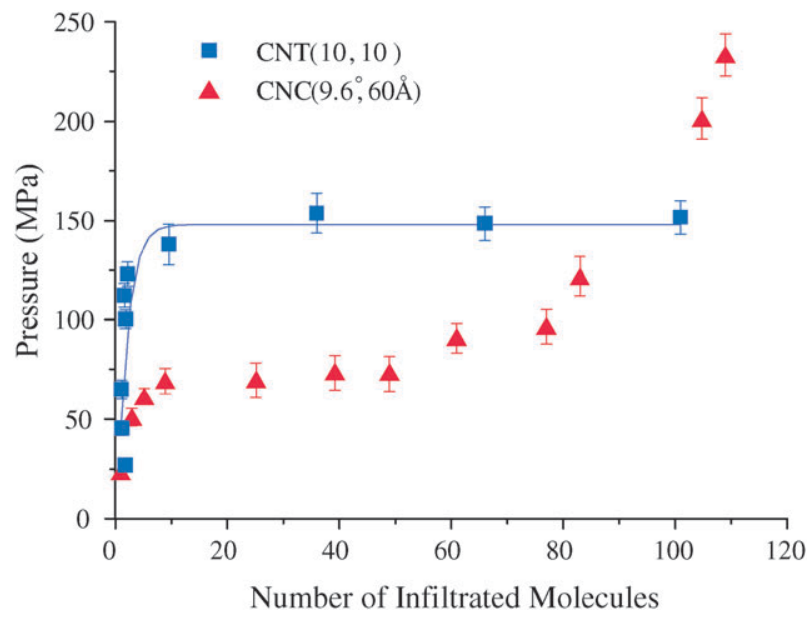

Fig. 3 The number of infiltrated water molecules in response to the applied pressure. The curve for CNT features a plateau at a critical pressure $\left(P_{\text {in }}\right)$, while that for $\mathrm{CNC}$ increases nonlinearly. The first and second indices of the $\mathrm{CNC}$ show its $\alpha$ and $H$, respectively.

molecules in response to the applied pressure for a $(10,10)$ armchair nanotube and a nanocone with $\alpha=9.6^{\circ}$, both of which expel water under ambient conditions. For the nanotube, the infiltration behavior follows the classical Young's relation, i.e. infiltration becomes spontaneous after a critical pressure, $P_{\text {in }}$, is attained. By contrast, in the nanocone, the pressure keeps increasing in a nonlinear fashion due to the varying pore size.

In order to more systematically investigate both spontaneous and pressure-assisted infiltration behaviors, in Fig. 4 we plot the relationship of pressure versus the pore diameter accessible to the infiltrated water front, as $\alpha$ is varied. The result effectively reveals the combined effects of wall inclination and pore size variation on the nanofluid infiltration (while the variation of $H$ is found to have negligible effect). First, for all nanocones studied in this paper, the pressure required for sustaining infiltration increases as the pore size is reduced, which is similar to the trend of the nanotube ( $\alpha=0^{\circ}$, solid square symbols in Fig. 4). Second, enlarging the apex angle always leads to lowered pressure for any given pore size. Third and most interestingly, when the apex angle is large, the pressure-pore size curve tends to intersect with the $P=0$ axis, reflecting the unusual mechanism transition

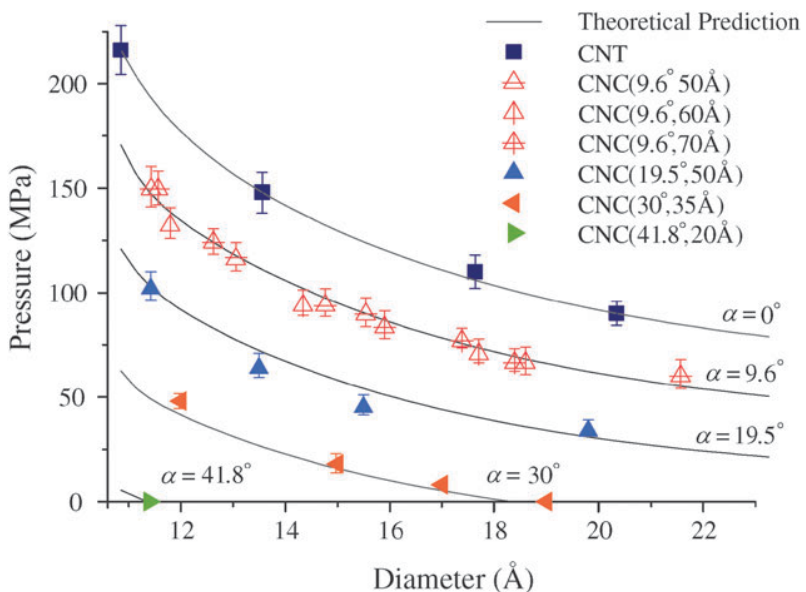

Fig. 4 The pressure-pore size relationship which depicts the external pressure required to infiltrate water molecules to a certain pore diameter accessible by the water front. The relationship is shown to be insensitive to the cone height, $H$. All symbols are from MD simulation while the lines are from the theoretical Young's relation (enriched with the nanoscale size effects). For nanotubes (square solid symbols) $P_{\text {in }}$ is uniquely identified according to the diameter. For each nanocone, the pressure is dependent on the infiltration depth (or volume, see Fig. 3) and therefore the pore size reached by the water front. The critical pore sizes identified for spontaneous infiltration in Fig. 2, 11.4 $\AA$ and $19 \AA$, are marked by the two symbols lying on the axis of $P=0$.

(namely, spontaneous infiltration followed by pressureassisted infiltration) observed for blunt nanocones. These interesting phenomena can be explained only by classical theories enriched with the size effects unique to the nanoscale, elaborated below.

From the energy point of view, there are two terms competing during the process of fluid infiltration into coneshaped nanopores, one associated with the liquid-solid interface and the other related to the liquid-vacuum interface (inset of Fig. 5). Denoting the surface energy per unit area of these two interfaces as $\gamma_{\mathrm{LS}}$ and $\gamma_{\mathrm{LV}}$, respectively, both of them

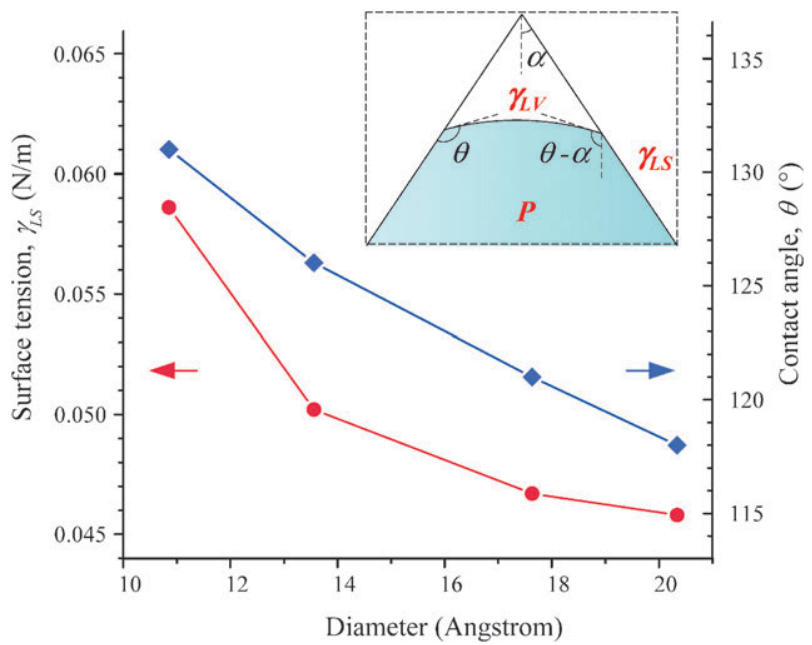

Fig. 5 The size effects of surface tension and contact angle involved in nanofluid infiltration. The inset shows a schematic illustration of the infiltration into cone-shaped nanopores. 
are positive given the hydrophobic solid phase, indicating that the expansion of such interfaces is energetically unfavorable. Thus, as the water front advances in a conical nanopore, the reduction of the liquid-vacuum interface energy must, to some extent, offset the energy increase due to the expanding liquid-solid interface. In this competition, the apex angle is a critical variable as it determines the relative shrinkage/ expansion rates of the two interfaces. When $\alpha$ is large enough, the reduction of the liquid-vacuum interface energy dominates, making the spontaneous infiltration energetically favorable. Likewise, pressure-assisted infiltration is easier in blunt nanocones.

Mathematically, the equilibrium of a meniscus inside a nanocone should take the form of $P=-4 \gamma_{\mathrm{LV}} \cos (\theta-\alpha) / D$, known as the Young's relation, where $\theta$ denotes the contact angle (between $90^{\circ}$ and $180^{\circ}$ for hydrophobic solids, inset of Fig. 5). Note that $\alpha$ largely determines the shape of the meniscus and the associated infiltration mechanism; a positive pressure is needed when $\theta-\alpha>90^{\circ}$, and spontaneous infiltration occurs when $\theta-\alpha \leq 90^{\circ}$. This Young's relation qualitatively matches the trend observed in Fig. 4: first, $P$ is inversely proportional to $D$; second, in the case of pressure-assisted infiltration, larger $\alpha$ results in lowered pressure for any given $D$.

Despite these qualitative matches, one critical phenomenon remains unexplained. That is, for a given combination of liquid and solid, once the cone apex angle $2 \alpha$ is specified and the contact angle $\theta$ is taken as that between the two bulk phases (and thus a constant), according to the Young's relation, the infiltration should be either spontaneous or pressure-assisted, and no transition like that observed for blunt nanocones should occur. This infers that the contact angle, $\theta$ must be size-dependent at the nanoscale. Such a size effect echoes the fact that, as the water front advances in a cone-shaped nanopore, both the liquid-vacuum interface and liquid-solid interfaces undergo substantial geometrical changes, leading to the size effects of interface energies and therefore the size effect of the contact angle.

In order to quantify the size effects involved in the current investigation, we first examine the infiltration into nanotubes of various characteristic sizes theoretically. The Young's relation becomes $P=P_{\text {in }}=-4 \gamma_{\mathrm{LS}} / D$, by letting $\alpha=0^{\circ}$ and using the equilibrium relationship, $\gamma_{\mathrm{LS}}=\gamma_{\mathrm{LV}} \cos \theta$. Based on MD simulation results (solid square symbols in Fig. 4), $\gamma_{\mathrm{LS}}$ can be obtained as a function of the tube size, $D$, plotted in Fig. 5. Such variation results from the size effects of both the liquid-solid binding energy and the surface energy of free-standing water droplets.

For nanocones, the Young's relation can be rewritten as $P=-4 \gamma_{\mathrm{LS}} \cos (\theta-\alpha) / D \cos \theta$; with the term $\gamma_{\mathrm{LS}}(D)$ calibrated above, by fitting the MD simulations in Fig. 4, one can obtain the size-dependent values of $\theta$ which are also plotted in Fig. 5. The result reflects a significant trend of increasing contact angle with decreasing size, which qualitatively matches the size effects of contact angle of water droplet on flat graphene. ${ }^{9}$ Incorporating these size effects, the resulting theoretical predictions (the size effect-enriched Young's relation where both $\gamma_{\text {LS }}$ and $\theta$ depend on $D$ ) shown in Fig. 4 (thin lines) match the MD simulation results quite well. Moreover, such size effects are identified as the mechanism responsible for the transition between spontaneous and pressure-assisted infiltration in blunt nanocones.

\section{Conclusion}

To summarize, we carried out a MD study on the fundamental behaviors of water infiltration into hydrophobic cone-shaped nanopores. The main findings include: (1) under ambient conditions, the applied pressure needs to be higher than a critical infiltration pressure such that water molecules may flow into a nanotube, and such infiltration pressure is higher for smaller tube. (2) Under ambient conditions, when the apex angle of a nanocone is large, spontaneous infiltration initiates despite the hydrophobic nature of the solid phase; however, water molecules can only access a particular pore size and cannot wet the entire nanocone. This implies an interesting transition between the spontaneous infiltration (nominally hydrophilic) and pressure-assisted infiltration (nominally hydrophobic) mechanisms. Furthermore, with the same blunt cone angle, a long nanocone and a short nanocone (with a pore opening smaller than the critical pore size that water can access under ambient conditions) may exhibit distinct wetting properties. (3) Further infiltration has to be assisted by an external pressure, and a higher pressure is required such that the water front may access a smaller pore size (i.e. advance further) in the nanocone. (4) Enlarging the apex angle always leads to lowered pressure to reach the same pore size and therefore easier infiltration. These phenomena can be explained by using the Young's relation enriched with nanoscale size effects, where both the liquid-solid interface tension and contact angle depend on the characteristic length. It is found that both the surface tension and contact angle increase as the size is reduced. The unique mechanisms revealed in this paper may contribute to the understanding and prediction of nanofluid infiltration into nanopores with more general cross-sections (slit-shaped, etc.), as well as help to design nanostructured surfaces with hydrophobichydrophilic (or wetting-nonwetting) transition capabilities.

\section{Acknowledgements}

The work is supported by NSF under Grant No. CMMI0643726 and CMS-0409521. L.L. acknowledges the support of the Founder's Prize, through the American Academy of Mechanics, sponsored by the Robert M. and Mary Haythornthwaite Foundation.

\section{References}

1 D. Mattia and Y. Gogotsi, Microfluid. Nanofluid., 2008, 5, 289-305.

2 R. B. Schoch, J. Y. Han and P. Renaud, Rev. Mod. Phys., 2008, 80, 839-883.

3 M. Whitby and N. Quirke, Nat. Nanotechnol., 2007, 2, 87-94.

4 F. Fornasiero, H. G. Park, J. K. Holt, M. Stadermann, C. P. Grigoropoulos, A. Noy and O. Bakajin, Proc. Natl. Acad. Sci. U. S. A., 2008, 105, 17250-17255.

5 X. Chen, F. B. Surani, X. Kong, V. K. Punyamurtula and Y. Qiao, Appl. Phys. Lett., 2006, 89, 241918.

6 S. Guenes and N. S. Sariciftci, Inorg. Chim. Acta, 2008, 361, $581-588$ 
7 R. E. Gyurcsanyi, TrAC, Trends Anal. Chem., 2008, 27, 627.

8 K. Healy, B. Schiedt and A. P. Morrison, Nanomedicine, 2007, 2, 875-897.

9 T. Werder, J. H. Walther, R. L. Jaffe, T. Halicioglu and P. Koumoutsakos, J. Phys. Chem. B, 2003, 107, 1345-1352.

10 G. Hummer, J. C. Rasaiah and J. P. Noworyta, Nature, 2001, 414, 188-190.

11 Y. Qiao, G. X. Cao and X. Chen, J. Am. Chem. Soc., 2007, 129, 2355-2359.

12 L. Liu, Y. Qiao and X. Chen, Appl. Phys. Lett., 2008, 92, 101927.

13 S. Joseph and N. R. Aluru, Nano Lett., 2008, 8, 452-458.

14 Y. Qiao, L. Liu and X. Chen, Nano Lett., 2009, 9, 984.

15 L. Liu and X. Chen, J. Phys. Chem. B, 2009, 113, 6468.

16 H. Daiguji, P. Yang and A. Majumdar, Nano Lett., 2004, 4, 137-142.

17 L. Liu, X. Chen, W. Lu and Y. Qiao, Phys. Rev. Lett., 2009, 102, 184501 .

18 M. Majumder, N. Chopra, R. Andrews and B. J. Hinds, Nature, 2005, 438, 44- 44

19 G. Hummer, J. G. Rasalah and J. P. Noworyta, Nature, 2001, 414, 188-190.

20 G. Cao, Y. Qiao, Q. Zhou and X. Chen, Philos. Mag. Lett., 2008, 88, 371.

21 G. Cao, Y. Qiao, Q. Zhou and X. Chen, Mol. Simul., 2008, 34, 1267.

22 J. Goldsmith and C. C. Martens, Phys. Chem. Chem. Phys., 2009, 11, 528-533.
23 C. C. Harrell, Y. Choi, L. P. Horne, L. A. Baker, Z. S. Siwy and C. R. Martin, Langmuir, 2006, 22, 10837-10843.

24 M. R. Powell, M. Sullivan, I. Vlassiouk, D. Constantin, O. Sudre, C. C. Martens, R. S. Eisenberg and Z. S. Siwy, Nat. Nanotechnol., 2008, 3, 51-57.

25 M. L. Kovarik and S. C. Jacobson, Anal. Chem., 2008, 80, 657-664.

26 J. Y. Jung, H. S. Oh, D. K. Lee, K. Bin Choi, S. K. Dong and H. Y. Kwak, J. Micromech. Microeng., 2008, 18, 7.

27 R. Shuttleworth and G. L. J. Bailey, Discuss. Faraday Soc., 1948, 3 , 16.

28 Y. Tsori, Langmuir, 2006, 22, 8860-8863.

29 X. Chen, G. X. Cao, A. J. Han, V. K. Punyamurtula, L. Liu, P. J. Culligan, T. Kim and Y. Qiao, Nano Lett., 2008, 8, 2988-2992.

30 L. Y. Meng, Q. K. Li and Z. G. Shuai, J. Chem. Phys., 2008, 128, 7.

31 S. Plimpton, J. Comput. Phys., 1995, 117, 1.

32 L. Y. Cao, L. Laim, C. Y. Ni, B. Nabet and J. E. Spanier, J. Am. Chem. Soc., 2005, 127, 13782-13783.

33 H. J. C. Berendsen, J. R. Grigera and T. P. Straatsma, J. Phys. Chem., 1987, 91, 6269.

34 M. J. Bojan and W. A. Steele, Langmuir, 1987, 3, 1123-1127.

35 F. A. Baum, L. O. Orlenko, K. P. Stanyukovich, V. P. Chelyshev and B. I. Shekhter, Physics of Explosion, 2nd edn, 1975, Nauka, Moscow. 